\title{
Latanoprost ophthalmic solution in the treatment of open angle glaucoma or raised intraocular pressure: a review
}

\author{
Andrea Russo \\ Ivano Riva \\ Teodoro Pizzolante \\ Federico Noto \\ Luciano Quaranta \\ Cattedra di Malattie dell'Apparato \\ Visivo, Università degli studi di \\ Brescia, USVD "Centro per lo studio \\ del Glaucoma” Spedali Civili di \\ Brescia
}

\begin{abstract}
Latanoprost is a prostaglandin F2-alpha isopropyl ester prodrug which is rapidly hydrolyzed by esterases in the cornea to the biologically active latanoprost acid. When latanoprost is topically administered into the eye, the cornea seems to act like as a slow-release depot to the anterior segment. One hour after administration maximum concentration is found in the iris, followed by the anterior chamber and the ciliary body. Despite extensive research, controversy remains about the real mechanism of action of this drug. Immunohistochemical data have shown that the intraocular pressure (IOP) reduction with topical prostaglandin F2-alpha is associated with a reduction of collagens within the uveoscleral outflow pathway. Evidence from several experimental and clinical studies suggests that latanoprost is a valuable addition first-line treatment alternatives for glaucoma, ocular hypertension and even angle-closure glaucoma. Strong points are its efficacy, which is demonstrated to be higher than that of brimonidine, dorzolamide and timolol with fewer systemic adverse effects; a convenient administration schedule; and the IOP-controlling pattern, which is relatively flat compared with timolol and dorzolamide, and enables better control in glaucoma progression, since large fluctuations may be associated with the risk of developing glaucoma in untreated ocular hypertensive subjects.
\end{abstract}

Keywords: latanoprost, intraocular pressure, glaucoma, ocular hypertension

\section{Introduction}

Glaucoma is the second leading cause of irreversible vision loss in the world, responsible for approximately $15 \%$ of blindness worldwide, and it is estimated that 6.7 million of the 67 million people affected by glaucoma worldwide are bilaterally blind (Quigley 1996). Glaucoma is a family of diseases not exactly defined by a specific intraocular pressure (IOP), but rather as an optic neuropathy that can occur with any IOP, depending on the susceptibility of the optic nerve.

Optic nerve damage is indeed not always related to a high IOP value: some patients show optic nerve damage with a relatively low IOP (normal-tension glaucoma), while others with persistent and pronounced IOP elevation (ocular hypertension) may never show such damage. These observations suggest that additional factors, such as vascular abnormalities and perfusion pressure, may play an important role in glaucomatous damage progression (Quaranta et al 1994).

Several variants of glaucoma exist, classified according to their etiology (ie, congenital, primary or secondary), but the main types are open-angle glaucoma (the most prevalent form in Caucasian and African populations) and angle-closure glaucoma (the most prevalent form among Asian and Eskimo populations).

Glaucoma incidence rises significantly with age: population studies show a dramatic rise in its prevalence after the age of 40. Furthermore, only one half of the patients have been diagnosed (Wensor et al 1998). 
Despite significant surgical and laser parasurgical progress, lowering IOP with topical drugs remains the initial and probably main treatment for most patients, including those with normal-tension glaucoma or ocular hypertension.

Latanoprost was introduced in the US in 1996 as the first prostaglandin-based IOP-lowering medication, receiving Food and Drug Administration approval between August 2000 and March 2001.

\section{Mechanism of action}

Latanoprost is a prostaglandin F2-alpha isopropyl ester prodrug (17-phenyl substituted PGF2-alpha), which is rapidly hydrolyzed by esterases in the cornea to a biologically active latanoprost acid; this undergoes a first metabolization passage in the liver (mainly though a b-oxidation process) before being excreted into urines and feces; the main metabolite in urines and feces was identified as the 1,2-dinor acid of latanoprost; furthermore, a more polar fraction from urine was identified as the 1,2,3,4-tetranor metabolite of the latanoprost acid.

When latanoprost is topically administered into the eye, the cornea seems to act like a slow-release depot to the anterior segment. One hour after administration maximum concentration is found in the iris, followed by the anterior chamber and the ciliary body. Elimination half-life from these tissues is 3 to 4 hours, although trace amounts can still be detected 24 hours after subministration. Analysis shows that latanoprost does not reach the posterior segment of the eye, being detectable only in more anterior tissues.

Despite extensive research, controversy remains about the real mechanism of action of this drug. Immunohistochemical data have shown that the IOP reduction with topical PGF2-alpha is associated with a reduction of collagens within the uveoscleral outflow pathway. The ciliary body contains several prostaglandin receptors (mainly FP and EP2 receptors), whose activation seems to stimulate a second messenger cascade for metalloproteinases synthesis (metalloproteinases are enzymes involved in extracellular matrix remodeling).

Ocklind (1998) treated human ciliary muscles with latanoprost for 1 to 2 days and then immunoassayed them against various extracellular matrix components and metalloproteinases. In response to latanoprost, collagen I, III, IV, fibronectin, laminin and hyaluronan were reduced, while metalloproteinases 2 and 3 were upregulated. These findings confirm a role for latanoprost in remodeling the extracellular matrix in order to allow an easier outflow of the aqueous humor through the ciliary muscle bundles.
This does not seem to be the only way latanoprost acts in lowering IOP: Sharif et al (2003) showed that prostaglandin analogs stimulate the prostaglandin FP receptors of human trabecular cells in vitro and this could increase matrix metalloproteinases within the meshwork and change the extracellular matrix, similar to the effect proposed for the uveoscleral pathway. Lim et al (2008) studied the effects of prostaglandin analogs on aqueous dynamics and found that they reduce the IOP by increasing the outflow either by the trabecular or the uveoscleral pathway, although which is the main pathway depends on the measurement technique used.

The most certain assumption is that latanoprost acts without significantly affecting the aqueous production rate, which could be an important property when used in combination (also fixed combinations) with other agents that reduce aqueous production (ie, $\beta$-blockers or carbonic anhydrase inhibitors).

\section{Efficacy}

Starting from the early years of treatment, clinical evidence showed that the best IOP control is achieved with one subministration of latanoprost per day rather than two (Nagasubramanian at al 1993). The reason for this is not fully clear, but is probably related to a down-regulation or a receptor subsensitivity that causes the loss of effect (Linden et al 1998).

After a single topical dose of latanoprost $0.005 \%$, IOP reduction is maximal within 8 to 12 hours and IOP remains below pretreatment level for at least 24 hours. In 24-hour IOP measurements, latanoprost administered once a day in the evening induces a constant IOP reduction, although the hypotensive effect seems to be greatest during the day (Quaranta et al 2006).

In patients with glaucoma or ocular hypertension (IOP $\geq 21$ ), a number of studies, between 1 and 12 months' treatment, report a reduced IOP level, from $22 \%$ to $39 \%$ (Perry et al 2003).

A meta-analysis of randomized clinical trials widely estimated the IOP reduction achieved by the most frequently prescribed glaucoma drugs and a placebo and pointed out that prostaglandin analogs are the most effective group for lowering IOP by monotherapy in primary open angle glaucoma (POAG) or ocular hypertensive patients, with a relative change of $-31 \%$ (at peak) and $-28 \%$ (at trough) for latanoprost (Van der Valk et al 2005).

After a long-term treatment, since latanoprost has no clinically significant effect on the permeability of the 
blood-aqueous barrier, IOP will return to pretreatment levels within a few weeks, indicating that latanoprost is safe for long-term treatments (Linden et al 1997).

\section{Comparative studies Timolol}

Several trials report that latanoprost is significantly more effective than timolol (timolol $0.5 \%$ administered twice daily) in reducing mean diurnal IOP and, while timolol maintains the same effects after 2 weeks of treatment, latanoprost has a further, significant, IOP reduction from 2 weeks to 6 months (Alm et al 1995; Camras et al 1996; Hedman et al 2000). This more consistent effect of latanoprost, in comparison with timolol, is more uniform and predominantly obtained during the night, probably because the rate of aqueous humor flow is lower during sleep and timolol, in contrast to latanoprost, acts on the production of humor flow rather than on the outflow (Reiss et al 1984).

In patients under timolol therapy, a significantly greater reduction in diurnal IOP can be achieved after addition of latanoprost to timolol monotherapy, although switching to latanoprost monotherapy could be attempted before starting combination treatment (Bucci 1999).

A latanoprost-timolol fixed combination, which has the advantage of a more convenient delivery system as well as providing a simple summing effect of the two constituents, is available on the market. Two studies show that this fixed combination of $0.005 \%$ latanoprost and $0.5 \%$ timolol, administered once daily in the morning for 6 months, is more effective in reducing IOP than the individual components alone (Pfeiffer et al 1996; Higginbotham et al 2002).

A meta-analysis by Webers et al (2006) pointed out that the concomitant use of $0.005 \%$ latanoprost and $0.5 \%$ timolol gave a change from baseline of $-6.0 \mathrm{mmHg}(-6.8$ to -5.2$)$ at the mean diurnal curve, while the fixed combination of $0.005 \%$ latanoprost and $0.5 \%$ timolol resulted in a mean change of $-3.0 \mathrm{mmHg}(-3.8$ to -2.2$)$ at the mean diurnal curve. This large difference can be explained by an omission of the timolol evening dose or by the different peak effect of latanoprost, timed 12 hours from the point of dosing (not 2 hours as for timolol), whereas the studies analyzed in this meta-analysis mostly measured IOP 0, 2 and 8 hours after the drops were used.

Pillunat in 2003 pointed out that latanoprost monotherapy is also a safe and effective alternative for those patients with open angle glaucoma under dual topical ocular hypotensive therapy, with a beta adrenergic receptor antagonist as one of the two components.

\section{Dorzolamide}

O'Donoghue et al (2000), in a study sample of 224 patients with open angle glaucoma or ocular hypertension, found that latanoprost was superior to dorzolamide 2\% (3 times a day) in reducing IOP, judged both from the effect on IOP at peak and trough and by the effect on diurnal IOP. Quaranta et al (2006) found a similar extent reduction at all time points between $10 \mathrm{PM}$ and $6 \mathrm{AM}$, which became a higher reduction in favor of latanoprost if the mean circadian IOP pressure value was considered.

Timolol-dorzolamide fixed combination (TDCF) administered twice daily gives a lower 24-hour pressure $(\mathrm{p}=0.004)$ and equally enhances 24-hour diastolic ocular perfusion pressure (DOPP) in previously untreated POAG patients compared with latanoprost (Quaranta et al 2008a). On the other hand, García Sanchez (2000), in the Spanish Latanoprost Study Group, found a $1.2 \mathrm{mmHg}$ statistically significant difference in IOP reduction in favor of latanoprost.

\section{Brimonidine}

Latanoprost, in a meta-analysis of randomized trials, was statistically more effective than brimonidine $0.2 \%$ twice daily as monotherapy in lowering IOP (Fung et al 2007). Furthermore, brimonidine is less effective than latanoprost in increasing DOPP (Quaranta et al 2006), since latanoprost does not affect systemic blood pressure as does brimonidine, which remains a selective $\alpha 2$-agonist and is associated with a higher rate of fatigue.

\section{Bimatoprost}

Although a little controversy still remains about the comparison of latanoprost with bimatoprost $0.03 \%$ (administered once daily), studies show that their IOP reduction is very similar similar. Simmons et al (2004) found greater IOP lowering with bimatoprost, ranging from 0 to $1.5 \mathrm{mmHg}$, which was not always statistically significant at all time-points but, according to the Early Manifest Glaucoma Trial, could be clinically significant in reducing the risk of progression in patients with glaucoma. In another study, Gandolfi et al (2001) reported that bimatoprost provided lower mean pressures than latanoprost at every time point throughout the study and was statistically superior in achieving low target pressures. In both studies mild conjunctival hyperemia was the most frequently reported adverse effect associated with either drug, but occurred more 
frequently with bimatoprost. In a recent study on normal tension glaucoma (NTG), Quaranta et al (2008b) showed that latanoprost and bimatoprost achieve a similar $16 \%$ extent in IOP lowering, which differs from the $32 \%$ reduction found in the Gandolfi study, probably because of the lower baseline pressure in NTG patients.

\section{Pilocarpine}

Latanoprost monotherapy is at least as effective as the twice-a-day fixed timolol-pilocarpine combination treatment in reducing mean diurnal IOP (Nordmann et al 2000).

Despite initial assessments in animals (Crawford et al 1987) showing that high concentrations of cholinergic agonists reduced drainage through uveoscleral pathway and attenuated the effects of a PGF2-alpha, Toris et al (2002) showed that while latanoprost increases uveoscleral outflow, pilocarpine increases outflow facility and pilocarpine does not block or attenuate the uveoscleral outflow effect of latanoprost, with a greater IOP reduction when these drugs are used in combination than when either drug is used alone. Kent et al (1999) assessed the order and timing of pilocarpine and latanoprost administration and found that they could significantly alter their hypotensive activity. Pilocarpine is most effective when administered 4 times a day and when the bedtime dose is administered 1 hour after the administration of latanoprost.

\section{Angle-closure glaucoma}

Some published data report Nd:YAG laser iridotomy as an effective therapy in widening the drainage angle and reducing elevated IOP in people with primary angle closure (Nolan et al 2002; Ahmed 2006). Despite this, medical therapy remains the best strategy available for those eyes still having uncontrolled IOP after iridotomy. Aung et al (2002) recruited 32 Asian patients with chronic primary angle-closure glaucoma (CACG), defined as glaucomatous optic neuropathy with a compatible visual field defect and at least 6 clock hours of synechial angle closure on gonioscopy. All patients had previous peripheral iridotomy (PI) with IOP $>21 \mathrm{mmHg}$ after PI and, after a washout period, were randomized to either receive timolol twice a day or latanoprost once a day. Compared with baseline, after 2 weeks of treatment the IOP was significantly reduced from a statistics point of view by $8.8 \pm 1.1 \mathrm{mmHg}(\mathrm{p}<0.001)$ in the latanoprost group, and by $5.7 \pm 0.9 \mathrm{mmHg}(\mathrm{p}<0.001)$ in the timolol group. The difference in IOP reduction between the two treatment groups was $3.1 \pm 1.5 \mathrm{mmHg}$ in favor of latanoprost $(p=0.04)$. A similar difference value $(3.0 \mathrm{mmHg})$ was found in a similar study by Chew et al in 2004 .
In an analogous population of patients, Chen et al (2007) studied the IOP-lowering effects of latanoprost versus bimatoprost and found that both drugs achieved a statistically significant IOP reducing effect compared with baseline, but there was no significant difference $(p=0.40)$ between the two groups. In a similar study carried out in 2006 , they also assessed latanoprost versus travoprost, finding a small but not significant $(\mathrm{p}=0.19)$ difference in IOP reduction $(0.7 \mathrm{mmHg})$ in favor of latanoprost.

In a comparison of latanoprost monotherapy and combined therapy of $0.5 \%$ timolol and $1 \%$ dorzolamide (unfixed combination) in CACG in Japanese patients, latanoprost monotherapy significantly lowered IOP compared with unfixed combination therapy of $0.5 \%$ timolol maleate and $1 \%$ dorzolamide hydrochloride (Sakai et al 2005).

An interesting study was carried out by Aung et al (2005) in order to evaluate any relationship between the configuration of the drainage angle and IOP-lowering efficacy of latanoprost in subjects with chronic CACG. The efficacy of latanoprost was not affected by the degree of angle narrowing or extent of synechial angle closure, a result that is probably correlated with the mechanism of action of latanoprost, which mainly facilitates the uveoscleral outflow rather than the trabecular one.

\section{Pigmentary glaucoma}

Pigmentary glaucoma (PG) is characterized by the liberation of pigment from the iris pigment epithelium and its deposition on various anterior segment structures including the trabecular meshwork, obstructing the physiological aqueous pathway. Thanks to latanoprost's uveoscleral remodeling, circumventing the trabecular outflow may be a viable alternative in pigmentary glaucoma management.

Mastropasqua et al (1999), in a trial with PG patients comparing $0.005 \%$ latanoprost applied once daily with $0.5 \%$ timolol applied twice daily, showed a greater hypotensive effect with latanoprost than timolol $(p<0.001)$; furthermore, after 3 months latanoprost achieved a persistent flattening of the diurnal IOP curve.

\section{Steroid-induced glaucoma}

The ocular or systemic administration of glucocorticoid can increase IOP, depending on individual responsiveness, by increasing the aqueous outflow resistance via morphological and chemical changes in the trabecular meshwork. Furthermore it provides a number of modifications in protein expression, cytoskeletal organization, extracellular matrix deposition, cell shape and immunological cells function 
(Clark et al 1995), although the full mechanism is not yet fully understood.

Vetrugno et al (2000) assessed the effect of $0.005 \%$ latanoprost and $0.50 \%$ timolol for the treatment of steroid-induced ocular hypertension and found that both drugs provide a significant and stable IOP reduction, without any real efficacy difference between the two drugs. A similar result was also found by Gupta et al (2007) in an experimental model of steroid-induced glaucoma in rabbits, in which the latanoprost IOP reduction $(35.06 \%)$ was similar to that for timolol (34.21\%). Another study confirmed the efficacy of latanoprost in steroid-induced glaucoma, reporting a $28 \%$ pressure reduction (Scherer et al 2000).

\section{Effects on blood flow}

The effects of latanoprost on ocular circulation in normal or glaucomatous eyes have been widely investigated using different methods. Vetrugno et al (1998) evaluated the effect on pulsatile ocular blood flow (POBF) of latanoprost in 12 patients aged 37 to 48; choroidal perfusion was measured through Langham's POBF system during the first day of therapy and then after 7, 15, 30, 60, 90 and 180 days; POBF values increased up to $55.8 \%$ in the first day and then settled at $22.6 \%$ at the end of the study; timolol showed distinctly lower blood perfusion values.

A similar positive result was found by $\mathrm{Koz}$ et al (2007): using color Doppler ultrasound they found a statistically significant lowering of resistive index (RI) in the ophthalmic artery after 6 months of latanoprost therapy. However, when latanoprost is associated to timolol in a fixed combination, the blood flow seems to be affected, with a significant decrease in end-diastolic velocity and a significant increase in RI, measured in the posterior ciliary arteries (Martinez et al 2007).

Harris et al (2008), using Heidelberg Retinal Flowmetry and Color-Doppler imaging measurements (including peak systolic velocity and end diastolic velocities), found that latanoprost has mostly neutral effects on ocular circulation, heart rate and blood pressure in healthy subjects. This is in contrast with the results found by Gherghel et al (2008), which shows that latanoprost increases mean ocular perfusion pressure (MOPP) and improves ocular perfusion at the optic nerve head and retina levels, measured with Heidelberg Retina Flowmeter system.

DOPP assessment is interesting, since a low DOPP $(<55 \mathrm{mmHg})$ is associated with an increased prevalence and incidence of glaucoma (Quigley et al 2001; Leske et al 2002). Latanoprost, in glaucomatous patients, achieves the best
DOPP increase $(+5.7 \mathrm{mmHg})$ compared to baseline, followed by dorzolamide $(+5.2 \mathrm{mmHg})$ and timolol $(+2.3 \mathrm{mmHg})$ (Quaranta et al 2006).

Because normal tension glaucoma is supposed to have a reduced choroidal perfusion in its pathogenesis, evaluation of ocular perfusion is an interesting subject to assess. Zeitz et al (2005) showed no significant alteration in systolic and diastolic blood flow velocities in the short posterior ciliary artery, assessed by color Doppler imaging 3 to 5 weeks after initiation local latanoprost therapy with either latanoprost or bimatoprost.

\section{Adverse events}

Published data show that latanoprost is generally well tolerated and, in contrast to timolol, is associated with minimal systemic adverse effects (Patel et al 1996), which are mostly mild and reversible on treatment discontinuation.

The most frequent adverse ocular side event was mild conjunctival hyperemia, which is a common effect of topical prostaglandin treatment (Susanna et al 2001), usually occurring within the first 2 days of treatment and declining over time. Compared with bimatoprost, conjunctival hyperemia is similar or more common with bimatoprost, while a light headache is more frequent with latanoprost (Dubiner et al 2001; Gandolfi et al 2002).

Latanoprost induces increased iridial pigmentation in $11 \%$ to $23 \%$ patients during a 1 -year treatment. The highest incidence of induced pigmentation was seen in green-brown, yellow-brown and blue/grey-brown eyes. Irides, homogeneously blue, grey, green or brown, are seldom affected. Typically, a concentric increase of the iris pigmentation appears after 6 months of treatment (judged to be noticeable by the patient in about two thirds of the cases) and, after cessation of latanoprost, increased pigmentation persists in patients who have been followed for 2 years (Wistrand et al 1997). The pathogenesis of this is not completely understood, although a study showed that in vitro latanoprost induces tyrosinase activity, rather than increasing the mitotic index in any of the human melanoma lines studied. This suggests that the in vivo iris pigmentation side effect of latanoprost may not result from increased cell division, but from elevated tyrosinase activity (Dutkiewicz et al 2000).

Latanoprost-treated eyes may develop hypertrichosis and increased pigmentation in the region of treatment (Johnstone et al 1997). Main changes in eyelash appearance include increased number, length, thickness, curvature and pigmentation. An increased pigmentation of the eyelid and 
periocular skin has also been reported (Wand et al 2001; Inoue et al 2006).

Because latanoprost is prostaglandin-based it may cause ocular inflammation, although when patients develop anterior chamber reaction during latanoprost treatment it seems to be caused by a mere alteration of the blood-aqueous barrier and not by a real inflammatory process (Ardjomand et al 2000). An objective study of the aqueous flare comparing latanoprost with travoprost and bimatoprost showed that after 3 months the travoprost $(\mathrm{p}<0.013)$ and the bimatoprost groups ( $p<0.001)$ had less flare than the latanoprost group, an effect that persisted even after 6 months (Cellini et al 2004). This is probably explained by a lightly superior break of the blood-aqueous barrier induced by latanoprost.

Smith et al (1999) showed that a mild delayed uveitis with latanoprost treatment may develop rarely, while in patients with a uveitis history, a mild delayed exacerbation potentially may occur and IOP may not be decreased in active uveitis.

Clinically symptomatic and angiographically documented cystoid macular edema (CME) associated with the use of latanoprost in pseudophakic eyes after uncomplicated cataract surgery has been reported, but a causal relationship between CME and latanoprost has not yet been proved or disproved.

Benzalkonium chloride (BAK), the most commonly used ocular preservative, has a very important role in the main adverse events, such as conjunctival hyperemia, decreased tear turnover, tear film break-up time and infiltration of the cornea by inflammatory cells. BAK is retained in tissues and can be found up to 168 hours after a single drop of $0.01 \%$ BAK in rabbits. The half-life of BAK in corneal epithelium and conjunctival tissues is 20 hours. Furthermore, prolonged exposure to BAK causes indirect and direct toxic effects to the ocular surface, including infiltration by inflammatory cells, reduced cellular viability and compromised epithelial barrier function through increased corneal epithelial cell permeability (Baudouin et al 2007). The toxic effects of BAK are dose-dependent, with progressive, slow, cell growth arrest occurring at concentrations as low as $0.0001 \%$, more rapid apoptosis at $0.01 \%$, and immediate epithelial cell necrosis at higher concentrations of $0.05 \%$ (De Saint et al 2002).

Baudouin et al (2007) investigated, in vitro, prostaglandin solutions preserved with BAK and the BAK-free solution travoprost Z $0.004 \%$, evaluating their effects on cellular viability, membrane integrity, and apoptosis through microtitration fluorometric assays and flow cytometry. They found that latanoprost with BAK, travoprost with BAK, and BAK alone have significant cytotoxic effects on human conjunctiva-derived cells and are associated with apoptosis. These effects likely result from the use of BAK as a preservative (Baudouin et al 2007).

In an animal model (Kahook et al 2008) changes were compared in the number of goblet cells after chronic exposure to latanoprost preserved with $0.02 \%$ BAK eye drops (Xalatan ${ }^{\circledR}$; Pfizer, NY, USA), travoprost preserved with sofZia ${ }^{\circledR}$ eye drops (Travatan $Z^{\circledR}$; Alcon, Fort Worth, TX, USA), or preservative-free artificial tears. Goblet cells per high-power field were counted after 30 days of once-daily topical application: $2.21( \pm 0.40)$ in the latanoprost with BAK group, $6.02( \pm 1.20)$ in the travoprost with sofZia group, and $7.03( \pm 1.33)$ in the preservative-free artificial tear group. The number of goblet cells in the latanoprost with BAK group was significantly lower than in the other two groups $(\mathrm{p}=0.0001)$.

Another in vivo study, based on a subjective symptom score and various clinical tests, supported these in vitro findings, showing that eyedrops containing BAK were associated with approximately 2 -fold higher odds showing abnormal results on the lissamine green staining test (Leung et al 2008).

The development of ocular preservatives that provide a non-toxic alternative to BAK will likely become useful in the management of chronic diseases such as glaucoma.

\section{Persistency}

As previously reported, the recommended dosage of latanoprost is a single drop once a day. This, together with its proven efficacy and favorable tolerability, is probably one of the most important characteristics of this drug, which has led to its best compliance and persistency among glaucoma medications. In population-based persistency studies performed in different geographic regions of the US, latanoprost users exhibited the greatest persistency (versus $\beta$-blockers, betaxolol, carbonic anhydrase inhibitors and brimonidine), without discontinuing, switching or adding a new class of glaucoma therapy (Dasgupta et al 2002; Shaya et al 2002; Spooner et al 2002).

\section{Cost-effectiveness analysis}

An economic evaluation of latanoprost cost-effectiveness ratio should be considered, since latanoprost, like all other glaucoma therapies, is a chronic therapy. 
Prostaglandin analogs for ophthalmic use are more expensive than alternative agents for lowering IOP and thus they need a careful assessment of their effectiveness.

Lachaine et al (2008) used IOP reduction in millimeters of mercury (compared with baseline and the incidence of adverse events) as the effectiveness data of the most used glaucoma medications, and found that dorzolamide, compared with latanoprost, is not a cost-effective strategy. Latanoprost, compared with brimonidine, has provided a higher IOP reduction with an incremental cost-effectiveness ratio, but the additional IOP reduction with latanoprost was obtained at a higher cost than the average cost per millimeter of mercury reduction obtained with brimonidine. Compared with timolol, latanoprost had a positive incremental cost-effectiveness ratio (Lachaine et al 2008).

Payet et al (2008) assessed the cost-effectiveness of latanoprost versus travoprost as single agents for the treatment of glaucoma in France and pointed out that travoprost demonstrated a longer effectiveness profile than latanoprost, although travoprost was more expensive than latanoprost (additional 5-year costs for travoprost were $€ 51$ ). This can be explained by a smaller proportion of patients needing a new treatment, laser therapy or surgery in the travoprost group, compensating for the higher travoprost acquisition cost (Payet et al 2008).

The real cost-effectiveness ratio may change from one country to another. For instance, in the UK timolol is more cost-effective than latanoprost, whereas in Scandinavia latanoprost may be more cost-effective than timolol (Stewart et al 2007).

\section{Conclusions}

Evidence from reported studies suggests latanoprost to be a valuable addition to the first-line treatment alternatives for glaucoma, ocular hypertension and angle-closure glaucoma. Strong points are efficacy, which is demonstrated to be higher than that of brimonidine, dorzolamide and timolol with fewer systemic adverse effects; the convenient administration schedule; and the IOP-controlling pattern, which is relatively flat compared with timolol and dorzolamide and allows a better control in glaucoma progression, since big fluctuations may be associated with the risk of developing glaucoma in untreated ocular hypertensive subjects. Another important point is the favorable effect of latanoprost on blood flow, providing a higher diastolic ocular perfusion pressure and thus better protection of the retinal nerve cells.

\section{Disclosures}

The authors have no conflicts of interest to disclose.

\section{References}

Ahmed M. 2006. Management of intermittent angle closure glaucoma with Nd: YAG laser iridotomy as a primary procedure. J Coll Physicians Surg Pak, 16:764-7.

Alm A, et al. 1995. Effects on intraocular pressure and side effects of $0.005 \%$ latanoprost applied once daily, evening or morning. A comparison with timolol. Scandinavian Latanoprost Study Group. Ophthalmology, 102:1743-52.

Ardjomand N, et al. 2000. Synthesis pattern of matrix metalloproteinases (MMPs) and inhibitors (TIMPs) in human explant organ cultures after treatment with latanoprost and dexamethasone. Eye, 14:375-83.

Aung T, et al. 2005. Degree of angle closure and the intraocular pressure-lowering effect of latanoprost in subjects with chronic angle-closure glaucoma. Ophthalmology, 112:267-71.

Aung T, et al. 2000. Comparison of the intraocular pressure-lowering effect of latanoprost and timolol in patients with chronic angle closure glaucoma: a preliminary study. Ophthalmology, 107:1178-83.

Baudouin C, et al. 2007. In vitro studies of antiglaucomatous prostaglandin analogues: travoprost with and without benzalkonium chloride and preserved latanoprost. Invest Ophthalmol Vis Sci, 48:4123-8.

Bucci MG. 1999. Intraocular pressure-lowering effects of latanoprost monotherapy versus latanoprost or pilocarpine in combination with timolol: a randomized, observer-masked multicenter study in patients with open-angle glaucoma. Italian Latanoprost Study Group. J Glaucoma, 8:24-30.

Camras CB, et al. 1996. Comparison of latanoprost and timolol in patients with ocular hypertension and glaucoma. United States Latanoprost Study Group. Ophthalmology, 103:138-47.

Cellini M, et al. 2004. Prostaglandin analogs and blood-aqueous barrier integrity: a flare cell meter study. Ophthalmologica, 218:312-7.

Chen MJ, et al. 2007. Comparison of the effects of latanoprost and bimatoprost on intraocular pressure in chronic angle-closure glaucoma. J Ocul Pharmacol Ther, 23:559-66.

Chen MJ, et al. 2006. Comparison of the effects of latanoprost and travoprost on intraocular pressure in chronic angle-closure glaucoma. $J$ Ocul Pharmacol Ther, 22:449-54.

Chew PT, et al. 2004. Intraocular pressure-reducing effects and safety of latanoprost versus timolol in patients with chronic angle-closure glaucoma. Ophthalmology, 111:427-34.

Clark AF, et al. 1995. Steroids, ocular hypertension and glaucoma. J Glaucoma, 4:354-69.

Crawford K, et al. 1987. Pilocarpine antagonizes prostaglandin F2 alpha-induced ocular hypotension in monkeys. Evidence for enhancement of Uveoscleral outflow by prostaglandin F2 alpha. Arch Ophthalmol, 105:1112-6.

Dasgupta S, et al. 2002. Population-based persistency rates for topical glaucoma medications measured with pharmacy claims data. Am J Manag Care, 8(10 Supp1):S255-61.

De Saint Jean M, et al. 1999. Effects of benzalkonium chloride on growth and survival of Chang conjunctival cells. Invest Ophthalmol Vis Sci, 40:619-30.

DuBiner H, et al. 2001. Efficacy and safety of bimatoprost in patients with elevated intraocular pressure: a 30-day comparison with latanoprost. Surv Ophthalmol, 45(Suppl 4):S353-60.

Dutkiewicz R, et al. 2000. Effects of latanoprost on tyrosinase activity and mitotic index of cultured melanoma lines. Exp Eye Res, 70:563-9.

Fung AT, et al. 2007. Meta-analysis of randomised controller trials comparing latanoprost with brimonidine in the treatment of open-angle glaucoma, ocular hypertension or normal-tension glaucoma. Br J Ophthalmology, 91:62-8.

Gandolfi S, et al. 2001. Three-month comparison of bimatoprost and latanoprost in patients with glaucoma and ocular hypertension. Adv Ther, 18:110-21. 
García Sanchez J. 2000. Efficacy and side effects of latanoprost monotherapy compared to adding dorzolamide to timolol in patients with glaucoma and ocular hypertension - a three-month randomised study. Spanish Latanoprost Study Group. Eur J Ophthalmol, 10:198-204.

Gherghel D, et al. 2008. First-line therapy with latanoprost $0.005 \%$ results in improved ocular circulation in newly diagnosed primary open-angle glaucoma patients: a prospective, 6-month, open-label study. Eye, 22:363-9.

Gupta SK et al. 2007. Comparative efficacy of pilocarpine, timolol and latanoprost in experimental models of glaucoma. Methods Find Exp Clin Pharmacol, 29:665-71.

Harris A, et al. 2008. The effect of latanoprost on ocular blood flow. Int Ophthalmol, Feb 23. [Epub ahead of print].

Hedman K, et al. 2000. A pooled-data analysis of three randomized, double-masked, six-month clinical studies comparing the intraocular pressure reducing effect of latanoprost and timolol. Eur J Ophthalmol, 10:95-104.

Higginbotham EJ, et al. 2002. Latanoprost and timolol combination therapy vs monotherapy: one-year randomized trial. Arch Ophthalmol, 120:915-22.

Inoue K, et al. 2006. Adverse reaction after use of latanoprost in Japanese glaucoma patients. Nippon Ganka Gakkai Zasshi, 110:581-7.

Johnstone MA. 1997. Hypertrichosis and increased pigmentation of eyelashes and adjacent hair in the region of the ipsilateral eyelids of patients treated with unilateral topical latanoprost. Am J Ophthalmol, 124:544-7.

Kahook MY, et al. 2008. Quantitative analysis of conjunctival goblet cells after chronic application of topical drops. Adv Ther, 25:743-51.

Kent AR, et al. 1999. Interaction of pilocarpine with latanoprost in patients with glaucoma and ocular hypertension. J Glaucoma, 8:257-62.

Koz OG, et al. 2007. Comparison of the effects of travoprost, latanoprost and bimatoprost on ocular circulation: a 6-month clinical trial. Acta Ophthalmol Scand, 85:838-43.

Lachaine J, et al. 2008. Prostaglandin analogues for ophthalmic use: a cost-effectiveness analysis. Can J Ophthalmol, 43:33-41.

Leske MC, et al. 2002. Incident open-angle glaucoma and blood pressure. Arch Ophthalmol, 120:954-59.

Leung EW, et al. 2008. Prevalence of ocular surface disease in glaucoma patients. J Glaucoma, 17:350-5.

Linden C, et al. 1998. Latanoprost twice daily is less effective then once daily: indication of receptor subsensitivity? Curr Eye Res, 17:567-72.

Lindén C, et al. 1997. Effects on IOP restoration and blood-aqueous barrier after long-term treatment with latanoprost in open angle glaucoma and ocular hypertension. Br J Ophthalmol, 81:370-2.

Martinez A, et al. 2007. Retrobulbar haemodynamic effects of the latanoprost/timolol and the dorzolamide/timolol fixed combinations in newly diagnosed glaucoma patients. Int J Clin Pract, 61:815-25.

Mastropasqua L, et al. 1999. A 12-month, randomized, double-masked study comparing latanoprost with timolol in pigmentary glaucoma. Ophthalmology, 106:550-5.

Lim KS, et al. 2008. Mechanism of action of bimatoprost, latanoprost, and travoprost in healthy subjects. A crossover study. Ophthalmology, 115:790-5.

Nagasubramanian S, et al. 1993. Intraocular pressure-reducing effect of PhXA41 in ocular hypertension. Comparison of dose regimens. Ophthalmology, 100:1305-11.

Nolan WP. 2000. YAG laser iridotomy treatment for primary angle closure in east Asian eyes. Br J Ophthalmol, 84:1255-9.

Nordmann JP, et al. 2000. Comparison of the intraocular pressure lowering effect of latanoprost and a fixed combination of timolol-pilocarpine eye drops in patients insufficiently controlled with beta adrenergic antagonists. French Latanoprost Study Group, and the Swedish Latanoprost Study Group. Br J Ophthalmol, 84:181-5.

O'Donoghue EP, et al. 2000. A comparison of latanoprost and dorzolamide in patients with glaucoma and ocular hypertension: a 3 month, randomised study. Ireland Latanoprost Study Group. Br J Ophthalmol, 84:579-82.
Ocklind A. 1998. Effect of latanoprost on the extracellular matrix of the ciliary muscle. A study on cultured cells and tissue sections. Exp Eye Res, 67:179-91.

Patel SS, et al. 1996. Latanoprost. A review of its pharmacological properties, clinical efficacy and tolerability in the management of primary open-angle glaucoma and ocular hypertension. Drugs Aging, 9:363-78.

Payet S, et al. 2008. Assessment of the cost effectiveness of travoprost versus latanoprost as single agents for treatment of glaucoma in France. Clin Drug Investig, 28:183-98.

Perry MP, et al. 2003. Latanoprost. An update of its use in glaucoma and ocular hypertension. Drugs Aging, 20:597-630.

Pfeiffer N, et al. 2002. A comparison of the fixed combination of latanoprost and timolol with its individual components. Graefes Arch Clin Exp Ophthalmol, 240:893-9.

Pillunat LE, et al. 2003. Intraocular pressure after replacement of current dual therapy with latanoprost monotherapy in patients with open angle glaucoma. Br J Ophthalmol, 87:1492-6.

Quaranta L, et al. 2006. Effects of topical hypotensive drugs on circadian IOP, blood pressure, and calculated diastolic ocular perfusion pressure in patients with glaucoma. Invest Ophthalmol Vis Sci, 47:2917-23.

Quaranta L, et al. 2008a. Effects of the timolol-dorzolamide fixed combination and latanoprost on circadian diastolic ocular perfusion pressure in glaucoma. Invest Ophthalmol Vis Sci, 49:4226-31.

Quaranta L, Pizzolante, T, Riva I, et al. 2008b. Twenty-four-hour intraocular pressure and blood pressure levels with bimatoprost versus latanoprost in patients with normal-tension glaucoma. Br J Ophthalmol, 92:1227-31.

Quaranta L, et al. 1994. The effect of increased intraocular pressure on pulsatile ocular blood flow in low tension glaucoma. Surv Ophthalmol, 38(Suppl):S177-81; discussion S182.

Quigley HA. 1996. Number of people with glaucoma worldwide. $\mathrm{Br} J$ Ophthalmol, 80:389-93

Quigley HA, et al. 2001. The prevalence of glaucoma in a population-based of Hispanic subjects. Proyecto VER. Arch Ophthalmol, 119:1819-26.

Reiss GR, et al. 1984. Aqueous humor flow during sleep. Invest Ophthalmol Vis Sci, 25:776-8.

Sakai H, et al. 2005. Comparison of latanoprost monotherapy and combined therapy of $0.5 \%$ timolol and $1 \%$ dorzolamide in chronic primary angleclosure glaucoma (CACG) in Japanese patients. J Ocul Pharmacol Ther, 21:483-9.

Scherer WJ, et al. 2000. Effect of latanoprost on intraocular pressure in steroid-induced glaucoma. J Glaucoma, 9:179-82.

Sharif NA, et al. 2003. Human trabecular meshwork cell responses induced by bimatoprost, travoprost, unoprostone, and other FP prostaglandin receptor agonist analogues. Invest Ophthalmol Vis Sci, 44:715-21.

Shaya FT, et al. 2002. Discontinuation rates of topical glaucoma medications in a managed care population. Am J Manag Care, 8(10 Suppl): S271-7.

Simmons ST, et al. 2004. Bimatoprost versus latanoprost in lowering intraocular pressure in glaucoma and ocular hypertension: results from parallel-group comparison trials. Adv Ther, 21:247-62.

Smith SL, et al. 1999. Latanoprost $0.005 \%$ and anterior segment uveitis. Acta Ophthalmol Scand, 77:668-72.

Spooner JJ, et al. 2002. Rates of discontinuation and change of glaucoma therapy in a managed care setting. Am J Manag Care, 8(10 Suppl): S262-70.

Stewart WC, et al. 2007. Cost-effectiveness of latanoprost and timolol maleate for the treatment of glaucoma in Scandinavia and the United Kingdom, using a decision-analytic health economic model. Eye, Aug 24. [Epub ahead of print].

Susanna R Jr, et al. 2001. The pros and cons of different prostanoids in the medical management of glaucoma. Curr Opin Ophthalmol, 12:149-56.

Toris CB, et al. 2002. Latanoprost and cholinergic agonists in combination. Surv Ophthalmol, 47(Suppl 1):S141-7. 
Van der Valk R, et al. 2005 Intraocular pressure-lowering effects of all commonly used glaucoma drugs: a meta-analysis of randomized clinical trials. Ophthalmology, 112:1177-85.

Vetrugno M, et al. 1998. Latanoprost $0.005 \%$ in POAG: effects on IOP and ocular blood flow. Acta Ophthalmol Scand Suppl, (227):40-1.

Vetrugno M, et al. 2000. A randomized, comparative open-label study on the efficacy of latanoprost and timolol in steroid induced ocular hypertension after photorefractive keratectomy. Eur J Ophthalmol, 10:205-11.

Wand M, et al. 2001. Latanoprost and periocular skin color changes. Arch Ophthalmol, 119:614-5.
Webers CA, et al. 2007. Intraocular pressure-lowering effect of adding dorzolamide or latanoprost to timolol. a meta-analysis of randomized clinical trials. Ophthalmology, 114:40-6.

Wensor MD, et al. 1998. The prevalence of glaucoma in the Melbourne visual impairment project. Ophthalmology, 105:733-9.

Wistrand PJ, et al. 1997. The incidence and time-course of latanoprost-induced iridial pigmentation as a function of eye color. Surv Ophthalmol, 41(Suppl 2):S129-38.

Zeitz O, et al. 2005. Effects of glaucoma drugs on ocular hemodynamics in normal tension glaucoma: a randomized trial comparing bimatoprost and latanoprost with dorzolamide. BMC Ophthalmol, 5:6. 
